\title{
Mejora de la calidad en el consentimiento informado
}

\author{
María Jesús Gómez Ramos ${ }^{1}$, Francisco Miguel González Valverde²
}

Resumen: Objetivo: Aplicar un círculo de mejora al consentimiento informado para la transfusión de hemoderivados. Pacientes y Método: Durante 10 meses se incluyeron 577 pacientes intervenidos en el Hospital General Reina Sofía de Murcia. Seleccionamos 6 criterios relacionados con el cumplimiento de la normativa sobre transfusiones. Tras el análisis de calidad, se aplicaron medidas correctoras y se reevaluaron los criterios. Los datos se compararon con los estándares en ambos periodos y entre periodos. En la primera evaluación se obtuvo un pobre cumplimiento de los criterios. Basándonos en el análisis de factores asociados al incumplimiento y la priorización resultante del diagrama de Pareto, el plan de intervención se dividió en actividad docente y modificaciones organizativas. Resultados: Todos los criterios estaban por debajo del estándar $(\mathrm{p}<0,001)$. En la segunda evaluación el cumplimiento de todos los criterios mejoró significativamente respecto de la primera; aun así los resultados continúan, también significativamente, por debajo de los estándares establecidos. Conclusiones: El cumplimiento de los 6 criterios era mínimo al inicio del estudio; las medidas correctoras fueron adecuadas, puesto que mejoraron todos ellos en la segunda evaluación, pero es necesario insistir en estas medidas ya que continuamos por debajo del estándar establecido.

Palabras clave: gestión de calidad, mejora de calidad, consentimiento informado, transfusión de hemoderivados

\section{Enhancement of informed consent quality}

\begin{abstract}
Aim: To apply an enhancement circle for informed consent for transfusion of blood derivatives. Patients and Method: During 10 months 577 intervened patients were included in General Hospital Reina Sofia of Murcia, Spain. Six criteria were selected related to compliance with transfusion norms. After quality analysis, corrected measures were taken and the criteria were re evaluated. Data were compared with standards in both periods and between periods. In the first evaluation a poor compliance to criteria was observed. Based on the analysis of factors associated to lack of compliance and the resulting priorization from Pareto's diagram, the intervention plan was divided in teaching activity and management modifications. Results: all criteria were under the standard $(\mathrm{p}<0.001)$. In the second evaluation, compliance for all criteria was significantly enhance with respect to the first; but nevertheless, results continue, also significantly, under established standards. Conclusions: Compliance to the six criteria was minimum at the beginning of the study; corrected measures were adequate, since they enhance all of them in the second evaluation, but it is necessary to insist in these measures since we continue under the established standard.
\end{abstract}

Key Words: quality management, quality enhancement, informed consent, transfusion of blood derivatives

\section{Melhora da qualidade no consentimento informado}

Resumo: Objetivo: Aplicar um círculo de melhora ao consentimento informado para a transfusão de hemoderivados. Pacientes e Método: Durante 10 meses foram incluídos 577 pacientes internados no Hospital General Reina Sofía de Murcia. Selecionamos 6 critérios relacionados com o cumprimento da norma sobre transfusóes. Após a análise de qualidade, foram aplicadas medidas corretoras e se reavaliaram os critérios. Os dados foram comparados com os padrôes em ambos periodos e entre periodos. Na primeira avaliação se obteve um pobre cumprimento dos critérios. Baseando-nos na análise de fatores associados ao incumprimento e a priorização resultante do diagrama de Pareto, o plano de intervenção se dividiu em atividade docente e modificaçóes organizativas. Resultados: Todos os critérios estavam abaixo do padrão ( $\mathrm{p}<0,001$ ). Na segunda avaliaçáo o cumprimento de todos os critérios melhorou significativamente com respeito à primeira; ainda assim os resultados continuam, também significativamente, abaixo dos padrốes estabelecidos. Conclusões: O cumprimento dos 6 critérios era mínimo ao início do estudo; as medidas corretoras foram adequadas, posto que melhoraram todos eles na segunda avaliação, porém é necessário insistir nestas medidas já que continuamos abaixo do padrão estabelecido.

Palavras-chave: gestão de qualidade, melhora de qualidade, consentimento informado, transfusão de hemoderivados

\footnotetext{
${ }^{1}$ Servicio de Medicina Intensiva. Hospital General Universitario Reina Sofía de Murcia, Espańa

Correspondencia: migova@terra.es

${ }^{2}$ Profesor Asociado. Departamento de Cirugía. Universidad de Murcia, Espańa
} 


\section{Introducción. Problema detectado}

Los pacientes intervenidos quirúrgicamente presentan, por sus particulares características, un alto riesgo de sangrado durante su estancia hospitalaria, bien debido a pérdidas en la intervención o bien por complicaciones posoperatorias. Este es el motivo de que determinadas patologías tengan asignada una reserva de concentrados de hematíes previo a la intervención, tanto programada como urgente. Además, algunos pacientes con una evolución tórpida (sepsis, shock hipovólemico, etc.) precisan transfusión de otros productos hemoderivados (plasma, plaquetas...). Según los datos manejados por el Banco de Sangre de nuestro centro, el Servicio de Cirugía es el principal receptor de trasfusiones hospitalarias con 88 peticiones cursadas por un total de 248 unidades de hemoderivados (concentrados de hematíes) en el último año.

Desde enero de 2006 es obligatoria en nuestro hospital la obtención del consentimiento informado $(\mathrm{CI})$, previo y específico del paciente o de sus representantes legales, para la transfusión de hemoderivados (artículo15, R.D. 1088/05, de 16 de septiembre). De ello se deriva la necesidad de informar y obtener la firma de un documento aparte del consentimiento quirúrgico. Recientemente, la Comisión de Hemoderivados nos ha comunicado que el cumplimiento de esta normativa por parte del Servicio de Cirugía ha sido en este tiempo muy bajo. Este hecho constituye un "problema" de calidad dentro del servicio, por lo que se planteó la posibilidad de realizar un ciclo de mejora con el fin de conocer las causas que impiden el cumplimiento de la normativa y tratar de subsanar el defecto de calidad(1).

\section{Pacientes y método}

El servicio de Cirugía General y Aparato Digestivo del Hospital General Universitario "Reina Sofía" de Murcia tiene asignadas en la actualidad 56 camas. Presenta 1.390 ingresos/año que resultan el $22,41 \%$ de los ingresos hospitalarios. De ellos, un $56,3 \%$ ingresa por urgencias, siendo el resto ingresos programados para intervención quirúrgica. Aplicamos el círculo de mejora de calidad propuesto por Palmer, que incluye una primera fase de evaluación, análisis estadístico de los re- sultados y propuesta de medidas correctoras. Una vez aplicadas, se vuelve a evaluar con el fin de conocer los cambios producidos.

Criterios para evaluar la calidad

Teniendo en cuenta la naturaleza del defecto de calidad se establecieron 6 criterios:

\section{Criterio 1.}

Definición: Los pacientes hospitalizados a los que se les curse una solicitud para transfusión de hemoderivados (concentrados de hematíes, plasma fresco, plaquetas, crioprecipitados, etc.) tendrán en su historia clínica un consentimiento informado correctamente cumplimentado y firmado por el paciente o su representante legal.

\section{Exclusión:}

- Intervenciones de extrema urgencia. Dicho concepto se define como toda situación en la que las lesiones del paciente amenazan su supervivencia y una demora en administrar la transfusión necesaria puede redundar en un perjuicio irreparable.

- Ausencia de representante legal, designado por incapacidad reconocida del paciente (menor de edad o establecida judicialmente) o por incapacidad temporal (bajo nivel de conciencia $\mathrm{o}$ inconsciencia del paciente).

Justificación: Dado que en nuestro centro las situaciones de exclusión no son frecuentes (1/1000 pacientes atendidos por dicho servicio), debe primar el derecho a la salud frente al derecho a la información. En el caso de extrema urgencia, entre preservar la vida y la necesidad de autorización, prima preservar la vida. En el caso de ausencia de representante legal, si no es posible obtener autorización para la transfusión y se entiende que se encuentra indicada, vuelve a primar el derecho a restituir la salud.

Aclaración: Una vez superadas dichas situaciones, si se precisara una nueva transfusión vuelve a prevalecer la definición del criterio 1.

Dicho documento se obtendrá previo a la petición de la primera bolsa y será válido para todo 
el ingreso hospitalario. Solo se cumple el criterio si el documento incluye la etiqueta identificativa y el DNI del paciente, y va firmado por él o su representante.

Las excepciones se consideran cumplimiento del criterio. Estándar del criterio: 100\%

\section{Criterio 2}

Definición: Todos los pacientes que ingresen de forma programada desde consulta para intervención quirúrgica tendrán cumplimentado y firmado en su historia clínica el CI para transfusión de hemoderivados durante dicho ingreso, sea o no necesaria la transfusión posteriormente.

Aclaración: Solo se cumple el criterio si el documento incluye la etiqueta identificativa y el número del DNI del paciente, y va firmado por él o su representante.

Estándar del Criterio: 100\%

\section{Criterio 3.}

Definición: En todos los documentos estará claramente especificada la fecha en la que se firma el consentimiento.

Aclaración: Solo se cumple el criterio si el documento incluye todos estos datos.

Estándar del Criterio: 100\%

\section{Criterio 4.}

Definición: En todos los documentos estará claramente especificado el facultativo que informa al paciente y firma el consentimiento.

Aclaración: Solo se cumple el criterio si el documento incluye todos estos datos.

Estándar del Criterio: 100\%

\section{Criterio 5.}

Definición: En todos los documentos se encontrará de forma legible la firma del facultativo que informa al paciente y firma el consentimiento. Se considerará legible que se reconozca el nombre y/o primer apellido del facultativo o bien que, al verla, sea posible adjudicarla sin ningún género de duda a un facultativo concreto. No se admitirá como firma legible un trazo aislado.

Aclaración: Solo se cumple el criterio si el documento incluye todos estos datos.

Estándar del Criterio: 100\%

\section{Criterio 6.}

Definición: En todos los documentos estará claramente identificado el motivo de solicitud de la transfusión.

Aclaración: Solo se cumple el criterio si el documento incluye todos estos datos.

\section{Estándar del Criterio: 100\%}

Dimensión estudiada: Como única dimensión se ha seleccionado la calidad científico-técnica, ya que además de la dimensión más amplia y con más subcomponentes, es la más cercana a la actuación del profesional y aquella sobre la que más directa y fácilmente podemos actuar.

Tipos de datos: Los criterios seleccionados pueden estar ligados a datos de estructura, proceso o resultado. En este estudio los criterios que empleamos estaban referidos a datos de proceso, dado que las modificaciones van dirigidas a "lo que hacemos".

Unidades de estudio:

Receptores:

Criterio 1: Recopilamos el total de enfermos para los que se cursa una solicitud de transfusión de hemoderivados desde planta en el Servicio de Cirugía General y del Aparato Digestivo. Incluye tanto a pacientes con ingreso programado como a los procedentes de Urgencias.

Criterio 2: Pacientes ingresados de forma programada, es decir, que han pasado una consulta previa con Cirugía General, para intervención quirúrgica reglada, y por tanto susceptibles de transfusión de hemoderivados.

Criterios del 3 al 6: Todos los pacientes en los 
que se solicite transfusión de hemoderivados en el Servicio de Cirugía General y del Aparato Digestivo; se transfundan o no posteriormente dichos hemoderivados.

Proveedores: los médicos del Servicio de Cirugía General del Hospital Reina Sofía.

Proceso Evaluado: Presencia del consentimiento informado previo a la primera transfusión de hemoderivados realizada durante el ingreso de los pacientes estudiados.

Marco muestral:

El estudio se llevó a cabo durante 10 meses. Durante 2 meses se realizó la primera evaluación y a continuación se aplicaron las medidas correctoras durante otros dos meses. La segunda evaluación se prolongó durante 6 meses más.

Fuentes de datos:

En estos criterios, los datos se recogieron de la historia clínica de los pacientes, incluyéndose en ese momento algunas variables epidemiológicas. La información se recogió al final del proceso y se guardó por los investigadores para su posterior evaluación.

Las variables epidemiológicas se dividían en dos apartados:

- Datos del paciente: Nombre, número de historia, sexo, edad, fecha de ingreso, estancia y destino final del paciente.

- Datos del proceso quirúrgico: Diagnóstico, urgencia de la intervención, tipo de intervención, complicaciones (número y tipo), reintervenciones (número y tipo de proceso).

Muestreo de las unidades de estudio:

Se seleccionaron todos los pacientes a los que se solicitó transfusión de hemoderivados y a aquellos que ingresaron de forma programada para intervención quirúrgica, en el Servicio de Cirugía General y del Aparato Digestivo.

Tipo de evaluación:
A pesar de que los datos se recogieron durante el tiempo en que el paciente estuvo ingresado en el hospital, el análisis de los mismos se realizó de forma retrospectiva una vez finalizado el periodo de estudio. Asimismo, el programa de evaluación fue interno, recogiéndose los datos por los profesionales responsables de este trabajo.

\section{Medidas correctoras:}

En nuestro estudio partíamos de la información del Servicio de Hematología, que confirmaba que en los primeros meses de vigencia del Real Decreto existían muy pocos documentos de consentimiento informado firmados para las transfusiones de hemoderivados de este servicio y en las solicitudes de reserva de sangre de las intervenciones quirúrgicas programadas. Por ello, previo a la primera evaluación, se informó a todos los facultativos del servicio de la existencia del Real Decreto y la obligatoriedad de su cumplimiento. Además, tanto en la consulta como en la planta de cirugía, se suministraron los impresos de consentimiento informado. Una vez analizadas las causas hemos establecido unas medidas correctoras divididas en dos apartados: Docentes y Organizativas:

Actividad docente: Como información general, se realizaron charlas formativas con el personal sanitario (facultativos y enfermería). En el caso de los facultativos, además de explicar el texto del Real Decreto y la obligatoriedad de su cumplimiento, se informó del contenido del documento de consentimiento y se comunicaron las modificaciones organizativas que se iban a establecer sobre el consentimiento informado.

Con el personal de enfermería, nuevamente se indicó la obligatoriedad del Real Decreto y se hizo hincapié en el hecho de que, si bien el personal de enfermería no es responsable de informar al paciente sobre el proceso y posibles complicaciones de la transfusión de hemoderivados, el trabajo de un Servicio Hospitalario es una labor de equipo y la implicación de todas las partes llevará consigo un mayor cumplimiento de los criterios y, por tanto, una mejora en la calidad del servicio prestado.

De modo particular y atendiendo a dudas concretas del personal sanitario (facultativos y enfer- 
mería), en primer lugar se estableció un protocolo sobre la cumplimentación de los impresos:

- En el caso de pacientes procedentes de la consulta y que ingresan para cirugía programada, el cirujano responsable de la intervención, cuando compruebe que el consentimiento informado firmado para la intervención se encuentra en la historia clínica del paciente previamente a la cirugía, también corroborará la existencia del consentimiento para transfusión de hemoderivados. De faltar éste, lo facilitará al paciente, aclarará sus dudas y ambos lo firmarán.

- En el caso de la planta de hospitalización, cuando el facultativo indique la transfusión en el tratamiento, la enfermera comprobará que se ha pasado el consentimiento al paciente. En su defecto facilitará el documento al paciente para que este lo lea y posteriormente consulte sus dudas con el médico y ambos lo firmen. Es importante comprobar que el paciente firma la autorización y no - por error- la denegación de autorización o revocación del consentimiento.

Otro cambio concreto se centró en el rechazo de los profesionales ocasionado por la gran cantidad de impresos que se deben cumplimentar para la intervención programada de un paciente, formalizando el uso del sello médico con la información de los datos profesionales del cirujano sobre el que posteriormente estampará su firma.

Modificaciones organizativas: En primer lugar, el impreso de consentimiento informado, dado que el Servicio de Documentación del hospital se encuentra en proceso de crear un nuevo modelo unido a la solicitud de transfusión, se almacenaba en el mismo archivador que la solicitud de transfusión y grapada a esta para que fuera más fácil recordar su existencia. Esta medida es válida tanto en la planta como en la consulta y los quirófanos, ya que la petición de transfusión o de reserva de sangre es la misma y por tanto útil la medida recordatoria.

En segundo lugar, la información al paciente se daba en la consulta externa, junto con la de la intervención a practicar. Ambos documentos de consentimiento eran entonces firmados por el paciente y el médico, y recogidos en la historia clínica, conservando el paciente para sí una copia firmada por el facultativo

\section{Tratamiento de los datos:}

Los resultados de los criterios se expresan en forma de porcentaje de cumplimientos (estimación puntual), con un intervalo de confianza del 95\% (estimación del intervalo de confianza). La estimación del intervalo de confianza se realiza mediante la fórmula $\mathrm{i}=\mathrm{z}$ x Sp (error o desviación estándar), donde $\mathrm{z}$ para un intervalo de confianza del 95\% es de 1,96. La fórmula de cálculo para la proporción de cumplimiento de un criterio de calidad es $S p=\sqrt{ } p(1-p) / n$, donde $p$ es la estimación puntual de la proporción de cumplimiento, y n es el tamaño de la muestra utilizada

La diferencia entre los valores de cumplimiento de los diferentes criterios de calidad se expresa de forma absoluta (P2-P1) y de forma relativa P2-P1/100-P1; donde P2 es la proporción de cumplimiento de la segunda evaluación y P1 la proporción de cumplimiento de la primera evaluación.

Para establecer la significación de las diferencias halladas, los valores de los criterios se compararán con los estándares establecidos $\left(^{*}\right)$ y con los porcentajes de cumplimiento previos $\left({ }^{* *}\right)$, mediante el valor de Z (una cola) para la comparación de proporciones. Finalmente, se utilizará el diagrama de Pareto para analizar gráficamente la mejora conseguida.

(*) Z para la diferencia entre proporciones de 2 evaluaciones:

$\mathrm{Z}=(\mathrm{P} 2-\mathrm{P} 1) / \sqrt{\mathrm{P}}(1-\mathrm{P})(1 / \mathrm{n} 1+1 / \mathrm{n} 2)$

Donde P2 es la proporción de cumplimiento de la segunda evaluación; P1 la proporción de cumplimiento de la primera evaluación; P la proporción de cumplimientos conjunta de la primera y la segunda evaluación $\left(\mathrm{P}=\left(\mathrm{n}^{\mathrm{o}}\right.\right.$ cumplimientos $1^{\mathrm{a}}$ evaluación $+\mathrm{n}^{\mathrm{o}}$ cumplimientos $2^{\mathrm{a}}$ evaluación) / $(\mathrm{n} 1+\mathrm{n} 2)) ; \mathrm{n} 1$ el tamaño de la muestra de la primera evaluación y n2 el tamaño de la muestra de la segunda evaluación. 
(**) Z para la diferencia entre el estándar y las proporciones de cumplimientos de una evaluación:

\section{$\mathrm{Z}=\mathrm{P}-\mathrm{S} / \sqrt{ } S(1-S) / \mathrm{N}$}

Donde P es la proporción de cumplimiento de la evaluación comparada; $\mathrm{S}$ el estándar de calidad establecido; $\mathrm{N}$ el tamaño de la muestra evaluada.

\section{Resultados}

\section{Análisis de datos de la primera evaluación}

Dado que las muestras son distintas para los criterios dentro de cada evaluación, es preciso normalizar los datos en relación con las frecuencias absolutas de incumplimientos. Se ha homogeneizado la muestra para todos los criterios al valor de C1 (148) (tabla 1).

Tabla 1. Cumplimientos absolutos de cada periodo

\begin{tabular}{|l|l|l|l|l|}
\hline & $\begin{array}{l}\text { Cumplimiento } \\
1^{a} \text { ev (n=148 } \\
\text { salvo c2 }=57)\end{array}$ & $\begin{array}{l}\text { Cumplimiento } \\
1^{a} \text { ev ajustada } \\
a c 1=148\end{array}$ & $\begin{array}{l}\text { Cumpliminento } \\
2^{a} \text { evaluacion } \\
(n=429 \text { salvo } \\
c 2=174)\end{array}$ & $\begin{array}{l}\text { Cumplimiento } \\
2^{a} \text { ev ajustada } \\
\text { acl }=148\end{array}$ \\
\hline Criterio 1 & 39 & 39 & 167 & 57 \\
\hline Criterio 2 & 48 & 125 & 171 & 145 \\
\hline Criterio 3 & 140 & 140 & 420 & 144 \\
\hline Criterio 4 & 51 & 51 & 253 & 87 \\
\hline Criterio 5 & 47 & 47 & 210 & 72 \\
\hline Criterio 6 & 157 & 157 & 420 & 144 \\
\hline
\end{tabular}

Se analizaron el total $\mathrm{N}=148$ (programados = 57) de las historias que debían ser incluidas en la primera fase.

10 Estimación grado de cumplimiento de los criterios evaluados: En la primera evaluación se obtuvo un pobre cumplimiento de todos los criterios, lo que se traduce en una diferencia estadísticamente significativa con su estándar $(\mathrm{p}<0,001)$ (tabla 2). Los criterios más incumplidos fueron los relativos al número total de impresos rellenados respecto del total de solicitudes y a la firma legible del facultativo, por lo que fueron los que mayor cantidad de defectos de calidad acumularon (criterios 1 y 5).
Tabla 2. Estimación del grado de cumplimiento de los criterios ( $1^{\mathrm{a}}$ y $2^{\mathrm{a}}$ evaluación) $\mathrm{N}=148$

\begin{tabular}{|l|l|l|l|l|l|}
\hline \multicolumn{2}{|l|}{} & \multicolumn{2}{l|}{$1^{a}$ Evaluación } & \multicolumn{2}{l|}{$2^{a}$ Evaluación } \\
\hline Criterio & \multicolumn{2}{|l|}{$p 1$ (IC 95\%) } & \multicolumn{2}{l|}{ (IC 95\%) $^{2}$} & \\
\hline 1. & $\begin{array}{l}\text { Presencia CI } \\
\text { transfusión }\end{array}$ & 26 & $( \pm 7.03)$ & 39 & $( \pm 7.9)$ \\
\hline 2. & $\begin{array}{l}\text { Presencia CI posible } \\
\text { transfusión cirugia } \\
\text { programada }\end{array}$ & 85 & $( \pm 5.8)$ & 98 & $( \pm 2.2)$ \\
\hline 3. & Fecha & 95 & $( \pm 3.54)$ & 98 & $( \pm 2.2)$ \\
\hline 4. & $\begin{array}{l}\text { Identificación } \\
\text { facultativo }\end{array}$ & 35 & $( \pm 7.69)$ & 60 & $( \pm 8.1)$ \\
\hline 5. & Firma facultativo & 32 & $( \pm 7.54)$ & 49 & $( \pm 8.1)$ \\
\hline 6. & Motivo transfusión & 93 & $( \pm 4.04)$ & 98 & $( \pm 2.2)$ \\
\hline
\end{tabular}

$2^{\circ}$ Análisis de los defectos de calidad y priorización de la intervención: En el diagrama de Pareto de esta primera evaluación (figura 1 "Pareto antes” y tabla 4) se reflejó que más del $80 \%$ de los incumplimientos se encontraba en 3 criterios (C1,C5 y C4), sobre los cuales se centraron específicamente las medidas correctoras.

Figura 1. Gráfico de Pareto

\section{PARETO ANTES}

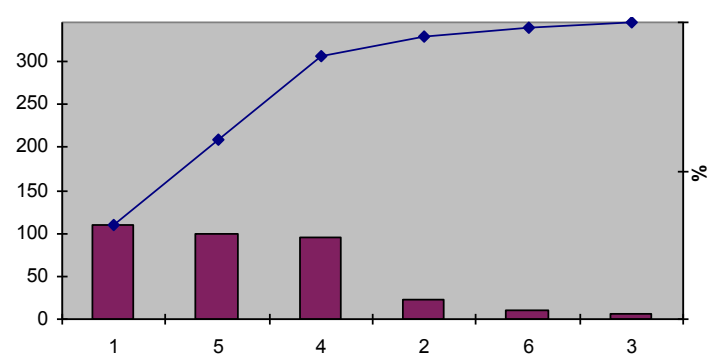

DESPUES

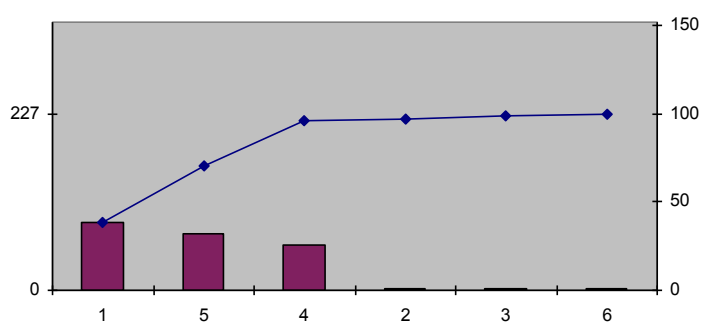




\section{Análisis de datos de la segunda evaluación}

Se analizaron el total $\mathrm{N}=429$ (programados = 174) de las historias que debían ser incluidas en la segunda fase. Los resultados se normalizaron respecto a $\mathrm{C} 1$ de primera evaluación, $\mathrm{n}=148$ (tabla 1).

$1^{\text {o }}$ Estimación grado de cumplimiento de los criterios evaluados: El análisis de los datos de esta evaluación respecto de la primera puso de manifiesto una mejora en todos los criterios, tanto los que se encontraban por debajo del estándar en la primera evaluación como de los que no (tabla 3). Esta mejoría fue estadísticamente significativa en los cinco de los seis criterios (excepción criterio 3). Respecto del estándar, todos los criterios continuaron por debajo y fue de forma estadísticamente significativa $(\mathrm{p}<0,001)$.

2o Análisis de los defectos de calidad: En esta segunda evaluación, el cumplimiento de todos los criterios ha mejorado respecto de la primera evaluación; todos, salvo el criterio 3, de forma estadísticamente significativa. Aun así, 3 criterios se encuentran, también significativamente, por debajo de los estándares establecidos (tabla 3).

Tabla 3. Estimación de la mejora conseguida

\begin{tabular}{|c|c|c|c|c|c|c|c|}
\hline \multirow{2}{*}{ है } & \multicolumn{2}{|c|}{ 1a Evaluación } & \multicolumn{2}{|c|}{$2^{a}$ Evaluación } & \multirow{2}{*}{$\begin{array}{l}\begin{array}{l}\text { Mejora } \\
\text { absoluta }\end{array} \\
p 2-p 1\end{array}$} & \multirow{2}{*}{$\begin{array}{l}\begin{array}{c}\text { Mejora } \\
\text { relativa }\end{array} \\
p 2-p 1 \\
1-p 1\end{array}$} & \multirow{2}{*}{$\begin{array}{l}\begin{array}{c}\text { Signifi- } \\
\text { cacion } \\
\text { estadistica }\end{array} \\
p\end{array}$} \\
\hline & $\begin{array}{l}p 1 \text { (IC } \\
95 \%)\end{array}$ & $\begin{array}{l}\Delta \text { d con el } \\
\text { Estandar }\end{array}$ & $\begin{array}{l}p 2 \text { (IC } \\
95 \%)\end{array}$ & $\begin{array}{l}\Delta \text { \& con el } \\
\text { Estanddar }\end{array}$ & & & \\
\hline 1 & $26( \pm 7.03)$ & $-91.35^{* *}$ & $39( \pm 7.9)$ & $-75,3^{* *}$ & 13.49 & 0.18 & $<0.01$ \\
\hline 2 & $85( \pm 5.83)$ & $-18.5^{* *}$ & $98( \pm 2.2)$ & $-2.46^{*}$ & 13.45 & 0.86 & $<0.01$ \\
\hline 3 & $95( \pm 3.54)$ & $-6.17^{7 * *}$ & $98( \pm 2.2)$ & $-2.46^{*}$ & 2.63 & 0.55 & n.s. \\
\hline 4 & $35( \pm 7.69)$ & $-80^{* *}$ & $60( \pm 8.1)$ & $-49.38^{* *}$ & 24.31 & 0.37 & $<0.001$ \\
\hline 5 & $32( \pm 7.54)$ & $-83.95 * *$ & $49( \pm 8.1)$ & $-62.96 * *$ & 16.52 & 0.25 & $<0.01$ \\
\hline 6 & $93( \pm 4.04)$ & $-8.64^{* *}$ & $98( \pm 2.2)$ & $-2.46^{*}$ & 4.66 & 0.68 & $<0.01$ \\
\hline
\end{tabular}

Tabla 4. Incumplimientos $1^{\text {a }}$ evaluación

\begin{tabular}{|l|l|l|l|}
\hline Criterio & $\begin{array}{l}\text { Fr Absoluta de Incum- } \\
\text { plimientos }\end{array}$ & $\begin{array}{l}\text { Fr Relativa de Incum- } \\
\text { plimientos (\%) }\end{array}$ & $\begin{array}{l}\text { Fr Relativa Acumulada } \\
\text { de Incumplimimen- } \\
\text { tos (\%) }\end{array}$ \\
\hline C1 & 110 & 31.79 & 31.79 \\
\hline C5 & 100 & 28.90 & 60.69 \\
\hline C4 & 96 & 27.74 & 88.43 \\
\hline C2 & 23 & 6.64 & 95.07 \\
\hline C6 & 10 & 2.86 & 97.93 \\
\hline C3 & 7 & 2.02 & 100 \\
\hline TOTAL & 346 & 100 & \\
\hline
\end{tabular}

Tabla 5. Incumplimientos $2^{\mathrm{a}}$ evaluación

\begin{tabular}{|l|l|l|l|}
\hline Criterio & $\begin{array}{l}\text { Fr Absoluta de Incum- } \\
\text { plimientos }\end{array}$ & $\begin{array}{l}\text { Fr Relativa de Incum- } \\
\text { plimientos (\%) }\end{array}$ & $\begin{array}{l}\text { Fr Relativa Acumulada } \\
\text { de Incumplimien- } \\
\text { tos (\%) }\end{array}$ \\
\hline C1 & 87 & 38.3 & 22.25 \\
\hline C5 & 73 & 32.2 & 43.98 \\
\hline C4 & 58 & 25.6 & 65.71 \\
\hline C2 & 3 & 1.3 & 84.38 \\
\hline C3 & 3 & 1.3 & 99.21 \\
\hline C6 & 3 & 1.3 & 100 \\
\hline TOTAL & 391 & 100 & \\
\hline
\end{tabular}

En el gráfico de Pareto (figura 1 "Pareto después" y tabla 5) se objetiva que nuevamente el $80 \%$ de los incumplimientos se centran en los criterios 1 , 5 y 4 relativos a que todos los pacientes a los que se les solicite una transfusión de hemoderivados tendrán un CI en su historia, y los relativos a la identificación y legibilidad de la firma del facultativo que solicita la transfusión.

\section{Discusión}

La información determina, en este contexto, la capacidad del paciente para ejercitar su derecho a disponer sobre su propio cuerpo. Este concepto de información al paciente para que tome sus propias decisiones es relativamente moderno $(2,3)$. La relaciones entre médico y paciente han venido marcadas por los principios morales de beneficencia proclamados por Aristóteles y así, durante siglos, el médico era el compendio del saber en esta parcela y por tanto el único con capacidad de juicio para decidir lo más conveniente para cada paciente en cada momento(4). Sin embargo, con la llegada de las nuevas tecnologías y el acceso a la información por parte de una amplía mayoría de la sociedad, se ha abandonado esa actitud paternalista en la relación médico-paciente, estableciéndose una de comunicación que permite a este último ejercer su derecho de decidir. Pero dentro de este marco ideal se producen fallos - a menudo importantes - en el modo, momento, lenguaje y capacidad del profesional para trasmitir esa información, aunque también en el paciente para comprenderla, asumirla e incluso para ser libre de decidir $(5,6)$.

Desde el punto de vista de la calidad es posible mejorar en este aspecto, tanto para mejorar la práctica asistencial como para satisfacer las expec- 
tativas de los usuarios. El ámbito de la sanidad y concretamente de los facultativos sanitarios es un marco muy adecuado para este tipo de mejoras: se trata de profesionales de alta cualificación, con titulación universitaria y formación especializada de posgrado; además, es un mundo en constante revisión a través de sesiones clínicas, publicaciones científicas, trabajo en equipo o comisiones clínicas(7). A pesar de ello, aún existen profesionales que se resisten a esta evaluación del trabajo diario; si bien cada vez más son los facultativos que entienden que la variabilidad de la práctica clínica, hay centros o incluso facultativos que ponen en duda la base científica y la calidad de nuestro trabajo(8). Este interés por la calidad de la práctica clínica ha generado multitud de oportunidades de mejora centradas sobre todo en los círculos de calidad, aunque existen además otros campos de mejora como la creación de protocolos de actuación, la satisfacción del usuario etc.(7).

El R.D. 1088/2005 regula las transfusiones de hemoderivados desde la solicitud hasta la comunicación de sus efectos adversos. Nuestro Servicio de Cirugía ya disponía de consentimientos informados concretos para cada patología susceptible de intervención quirúrgica, que incluían la posibilidad de transfusión de concentrados de hematíes durante la intervención o el posoperatorio. En el documento, este apartado estaba redactado sobre todo en el contexto de las objeciones morales y religiosas; sin embargo, según la legislación mencionada, es necesario un consentimiento propio, específico y que haga referencia a las complicaciones concretas de la transfusión de hemoderivados. Desde la comisión clínica correspondiente se dispusieron medidas para la implantación de este nuevo documento, informando a jefes de servicio y supervisores de enfermería de su entrada en vigor. Asimismo, desde la unidad de hemodonación se estableció no facilitar hemoderivados si la petición no se acompañaba del documento de consentimiento cumplimentado, salvo en caso de urgencia extrema.

A pesar de que estas medidas se iniciaron con dos meses de antelación, se nos comunicó que el Servicio de Cirugía General era el servicio con menor cumplimiento del RD, trascurrido un mes de su entrada en vigor. Ante estos resultados, en nuestro servicio advertimos una oportunidad de mejora, en primer lugar por un aumento de la calidad de nuestra practica clínica y, además, por la posibilidad de dar un primer paso para la mejora global de los consentimientos informados.

Los resultados de la primera evaluación corroboraron los datos aportados por la comisión clínica de hemoderivados: todos los criterios se encontraban por debajo del estándar establecido. En el caso del primer y segundo criterio, el bajo cumplimiento se podía explicar por tratarse de una primera medición sin implantación previa de medidas de mejora y ser un documento reciente, con lo que aún el impreso no se identificaba y obtenía fácilmente, ya que por su novedad podía no estar disponible en todas las consultas y/o plantas. En el resto de los criterios (del $3^{\circ}$ al $6^{\circ}$ ) no encontramos justificación, por no tratarse de una medida ajena a la práctica habitual en un servicio hospitalario, ya que en cualquier acto médico debe constar obligatoriamente la fecha en que se realiza, la identificación correcta del facultativo junto a su número de colegiado y su firma. Así pues, partíamos de un bajo cumplimiento de todos los criterios, especialmente en el caso del primero que lógicamente acumulaba el mayor porcentaje de incumplimientos y que era, por tanto, el más susceptible de cambios para mejorar.

La mayoría de las medidas encaminadas a incrementar el cumplimiento de los criterios fueron de tipo docente, centrándolas en informar a todo el equipo sanitario (facultativos y enfermería) del significado, indicaciones y responsabilidades de cada profesional en la cumplimentación del consentimiento, recalcando su carácter de documento legal y la necesidad de que se manejara como tal. Además, se establecieron estrategias que facilitaran al médico su acceso al documento, como grapar una copia a cada impreso de petición, atendiendo a las diferentes situaciones en las que puede ser necesaria la transfusión de hemoderivados (paciente ingresado, en urgencias o cirugía programada).

Por otro lado, se establecieron medidas organizativas para simplificar los trámites y que no fuese posible solicitar cualquier transfusión sin cumplimentar el documento. En el caso de los cuatro últimos criterios se recordó periódicamente, en la sesión clínica ordinaria, la necesidad de legitimar 
el documento, tanto con la fecha como con los datos correctos del facultativo informante.

En la reevaluación, tras dos meses de aplicación de las medidas correctoras, encontramos una mejora significativa en todos los criterios, especialmente en el primero. Este resultado podía ser esperable teniendo en cuenta el bajo nivel de cumplimiento de que partíamos. La percepción por los facultativos del CI como documento legal nos hace pensar que la extensión de su uso obedeció en parte a estrategias defensivas más que de fomento de la participación de los pacientes y de la calidad asistencial. Se tiende a disociarlo del proceso de interacción con el paciente. Una de las claves para mejorar la situación sería la desjudicialización de la relación médico-paciente, y la reflexión sobre los objetivos políticos que se persiguen con la implantación del consentimiento informado escrito. Aun así permanecemos por debajo de los estándares establecidos, de forma significativa, en los seis criterios analizados. En el análisis del gráfico de Pareto nuevamente el criterio 1 continúa siendo el punto sobre el más se debe incidir para continuar mejorando. Sin embargo, a la luz de estos resultados, consideramos que las medidas establecidas han sido muy acertadas y son el camino para continuar mejorando.

El propósito que nos ha guiado en este trabajo no ha sido otro que mejorar en un ámbito importante de nuestra actividad clínica. Podemos razonar que las deficiencias encontradas son en parte debidas a la falta de formación y reciclaje en estos contenidos, o una consecuencia de la excesiva demanda asistencial que impide atender como desearíamos a nuestros pacientes, pero no debemos permitir que esta realidad constituya un impedimento para tratar de mejorar. Es preciso desarrollar estrategias de comunicación que acerquen al paciente y al profesional de la medicina, perfeccionando y haciendo accesibles a la comprensión los documentos de consentimiento informado y, lo más importante, una relación médico-paciente satisfactoria (8-12). 


\section{Referencias}

1. Saturno PJ. Evaluación y mejora de la calidad en servicios de salud. Conceptos y métodos. Murcia: Consejería de Sanidad y Consumo de la Región de Murcia; 2000.

2. Ley 41/2002, de 14 de noviembre, básica reguladora de la autonomía del paciente y de derechos y obligaciones en materia de información y documentación clínica. BOE, 15 de noviembre de 2002.

3. Sánchez-Caro J. La ley sobre autonomía del paciente y su repercusión en las Comunidades Autónomas. Rev Adm Sanit 2003; 1: 189-202.

4. The Hastings Center. The Goals of Medicine. Setting New Priorities. Special Supplement. Hastings Center Report 1996; 26(6): S1-S27.

5. Collazo E. Consentimiento informado en la práctica médica. Fundamentos bioéticos y aspectos prácticos. Cir Esp 2002; 71(6): 319-324.

6. Simón Lorda P, Rodríguez Salvador JJ, Martínez Maroto A, López Pisa RMa , Júdez Gutiérrez J. La capacidad de los pacientes para tomar decisiones. Med Clin (Barc.) 2001; 117: 419-426.

7. Oteo Ochoa LA. Gestión clínica: análisis del entorno y desarrollo organizativo. En: Módulo 6. Desarrollos Organizativos de Gestión Clínica. III Curso Experto Universitario en Gestión de Unidades Clínicas. UNED. ENS, ISCIII. Madrid; 2001-2002.

8. González Valverde FM, Gómez Ramos MJ, Méndez Martínez M, Rodenas Moncada FJ. Evaluación y mejora del cumplimiento de la normativa sobre consentimiento informado para la transfusión de hemoderivados en un servicio de cirugía general. Rev Calidad Asistencial 2008; 23(3): 142-143.

9. Morales Cuenca G, Aguilar Jiménez J, Aguayo Albasini JL. Memoria de un servicio de Cirugía General y Aparato digestivo. Arch Cir Gen 2003 jul 31.

10. Baños Madrid R, Ramón Esparza T, Serrano Jiménez A, Alajarín Cervera M, Alberca de las Parras F, Molina Martínez J. Evaluación y mejora en la información de los pacientes que se realizan una endoscopia. An Med Interna (Madrid) 2005; 22: 413-418.

11. Montoya JA, Ballesteros AM, Fontcuberta J, García A, Santiago C, Moreno MA. Atención al paciente diabético en un área de salud: ciclo de mejora Rev Calidad Asistencial 2003; 18(4): 203-208.

12. Comisión nacional para la protección de los sujetos humanos de investigación biomédica y del comportamiento. Principios y guias éticas, para la protección de los sujetos humanos de investigación. USA; 18 de abril de 1979.

Recibido: 29 de mayo de 2011

Aceptado: 15 de agosto de 2011 